INDIAN JOURNAL OF PHYSICS

ISSN: 0973-1458 (print version); ISSN: 0974-9845 (electronic version); PUBLISHER: SPRINGER

Impact factor $=0.967$

Accepted April 11 2019

\title{
NUMERICAL STUDY OF SELF-SIMILAR NATURAL CONVECTION MASS TRANSFER FROM A ROTATING CONE IN ANISOTROPIC POROUS MEDIA WITH STEFAN BLOWING AND NAVIER SLIP
}

\author{
O. Anwar Bég ${ }^{1}$, M.J. Uddin², T. A. Bég3, A. Kadir ${ }^{4}$, MD. Shamshuddin5 ${ }^{*}$ \\ and Meisam Babaie ${ }^{6}$ \\ ${ }^{\mathbf{1 , 4}}$ Aeronautical \& Mechanical Engineering Department, School of Computing, Science and Engineering, Newton Building, \\ University of Salford, Manchester, M54WT, UK. \\ ${ }^{2}$ Department of Mathematics, University Sains-Malaysia, Malaysia. \\ ${ }^{3}$ Computational Mechanics and Renewable Energy, Dickenson Road, Manchester, M13, UK. \\ $\mathbf{5}^{*}$ Department of Mathematics, Vaagdevi College of Engineering, Warangal, Telangana, India. \\ ${ }^{6}$ Energy Sciences, Petroleum and Gas Engineering, University of Salford, Manchester, M54WT, UK. \\ *Corresponding author: shammaths@gmail.com; shamshuddin_md@vaagdevi.edu.in
}

\begin{abstract}
A mathematical model is presented for laminar, steady natural convection mass transfer in boundary layer flow from a rotating porous vertical cone in anisotropic high permeability porous media. The transformed boundary value problem is solved subject to prescribed surface and free stream boundary conditions with a MAPLE 17 shooting method. Validation with a Chebyshev spectral collocation method is included. The influence of tangential Darcy number, swirl Darcy number, Schmidt number, rotational parameter, momentum (velocity slip), mass slip and wall mass flux (transpiration) on the velocity and concentration distributions is evaluated in detail. The computations show that tangential and swirl velocities are enhanced generally with increasing permeability functions (i.e. Darcy parameters). Increasing spin velocity of the cone accelerates the tangential flow whereas it retards the swirl flow. An elevation in wall suction depresses both tangential and swirl flow. However, increasing injection generates acceleration in the tangential and swirl flow. With greater momentum (hydrodynamic) slip, both tangential and swirl flows are accelerated. Concentration values and Sherwood number function values are also enhanced with momentum slip, although this is only achieved for the case of wall injection. A substantial suppression in tangential velocity is induced with higher mass (solutal) slip effect for any value of injection parameter. Concentration is also depressed at the wall (cone surface) with an increase in mass slip parameter, irrespective of whether injection or suction is present. The model is relevant to spin coating operations in filtration media (in which swirling boundary layers can be controlled with porous media to deposit thin films on industrial components), flow control of mixing devices in distillation processes and also chromatographical analysis systems.
\end{abstract}

Keywords : Mass transfer, rotating cone, slip, anisotropic porous media, wall suction/injection.

PACS No. : 46.15.-X, 47.11. +j, 47.10. ab, 44.30. +v, 07.05. Tp.

\section{Introduction}

Mass transfer (species diffusion) with or without buoyancy forces is fundamental to many diverse procedures in modern chemical, environmental and biomedical engineering. It arises in catalytic packed-bed reactors [1], geological contaminant dispersion [2], oil spill penetration into stratified soils [3] and oxygen diffusion in neurological tissue [4]. The established methodology for simulating mass transfer is the Fickian law of mass diffusion. To simulate transport in porous media, a variety of methods have been employed to solve the Fickian diffusion equation in permeable systems ranging from drag-force formulations to percolation 
theory, in order to recreate the permeability (hydraulic conductivity) properties of such media. Ulson De Souza and Whitaker [5] used the volume-averaging method to simulate mass transfer in a packed-bed reactor, examining in detail the dispersion in the main fluid phase, internal diffusion of the reactant in the pores of the catalyst and surface reaction within the catalyst. Helmer et al. [6] studied unsteady water diffusion in tumors (diseased tissue) with a tortuous porous medium model. Cotta et al. [7] applied integral transforms to study a range of convective boundary layer mass transfer problems in permeable systems. Piquemal et al. [8] simulated species mobility in porous media with a dispersion-convection equation for the mobile fluid and a diffusion equation for the stagnant fluid, considering a cylindrical tube with stagnant pockets in its wall and also studied a stratified permeable medium. Vafai and Tien [9] conducted experiments and numerical studies of convective buoyancy-driven mass transfer in porous media with Brinkman friction and Forchheimer inertial drag effects, evaluating the time and space-averaged mass flux of a species in the porous medium. In these studies, the permeability of the material was considered to be isotropic i.e. permeability was assumed to be the same in any direction. However, in certain industrial filtration materials and foams and also invariably in geological systems, porous media are anisotropic. The variation in permeability in different directions can have a dramatic effect on transport phenomena and can influence, for example, the fate of contaminants, the rates of mass transfer on embedded body surfaces etc. Several articles have addressed anisotropic porous media hydrodynamics. Marcus [10] was among the first researchers to investigate anisotropic flows in porous media. He defined a directional permeability and conducted laboratory tests on carefully designed samples. Wang et al. [11] considered a variety of different structural models to analyze nonDarcy flow in anisotropic porous media surrounding the near-wellbore region of high-capacity gas and condensate reservoirs. They showed that as pore-scale anisotropic parameter is increased, there is a reduction in permeability components. Adams et al. [12] used a finite element method to simulate radial encroachment of a viscous liquid into a homogeneous, anisotropic porous medium, deriving effective permeabilities as functions of the principal permeabilities. Other studies [13-14] examined the topology of flow in three-dimensional nonstationary anisotropic heterogeneous porous media with a Monte Carlo simulator. Nakayama and Kuwahara [15] determined the permeability tensor for isothermal anisotropic porous media.

The flow from a rotating curved body is also of great interest in chemical engineering operations. The Coriolis forces which are generated by centrifugal fields encourage fluid to be drawn along the curved surface and significantly alter momentum diffusion rates. If species diffusion also occurs, mass transfer rates at the curved surface are also generally enhanced. Although many investigations have been reported on heat transfer from spinning bodies, relatively few investigations have considered mass transfer. Salzberg and Kezios [16] presented an early experimental study of mass-transfer by sublimation from the surface of a rotating naphthalene cone in an airstream. Newman [17] employed laminar boundary layer theory to determine the limiting rates of mass transfer to a rotating sphere at high Schmidt 
numbers. Smith and Colton [18] developed approximate solutions for mass transfer from a disk to a rotating fluid, observing that mass transfer is dominated by the outer-most zone of the disk owing to high transfer rate associated with the leading edge of the boundary layer, and furthermore that the species concentration field close to the axis of rotation extends to significant axial distances as a long slender plume. Ellison and Cornet [19] determined experimentally the mass transfer rates for oxygen diffusion to a disk rotating in aqueous sodium chloride solution and calculated average Sherwood numbers over an extensive range of Reynolds and Schmidt numbers. Laminar-turbulent transition in mass transfer from a rotating disk was investigated by Mohr and Newman [20]. Toren et al. [21] studied centrifugally-driven flow (due to a density gradient between the surface of an infinite disk and the ambient fluid) in rotating Von Karman mass transfer at high Schmidt number. They identified a linear Ekman layer driven by a buoyancy sublayer. Rashaida et al. [22] evaluated the laminar boundary layer mass transfer from a rotating disk to a Bingham non-Newtonian fluid, deriving a Sherwood number as a function of Reynolds number and dimensionless yield stress (Bingham number), and observing that higher Bingham number depresses wall mass transfer rates.

In recent years slip flows have also attracted the attention of engineers. These flows arise when the Navier no-slip boundary condition, a common approximation in viscous fluid dynamics, is not applicable. Slip may occur in the velocity field, thermal field ("thermal jump") or mass distribution (species concentration distribution), at a boundary. It arises in certain polymeric manufacturing processes as well as in rarefied gas dynamics in high speed flight. Theoretical models often use the Navier condition for momentum slip. Taamneh and Omari [23] examined computationally the slip-flow and heat transfer in non-Newtonian inelastic fluids in a porous medium micro-channel, using a Knudsen number to evaluate slip effects and showing that with greater Knudsen numbers, the wall shear stress is enhanced i.e. the flow is accelerated. Miguel [24] established several discrete regimes for slip flow in porous media including free molecular (ballistic) flows at very high Knudsen numbers. He also observed that for a slip-flow regime, the dimensionless permeability of the porous medium is dependent on the structure of the medium and exhibits a power-law increase with the Knudsen number for high porosities. Other slip flow transport phenomena have been studied by Khan et al. [25] for nanofluid heat and mass transfer, Bég et al. [26] for magnetohydrodynamic radiative convective flow, Wang [27] for Newtonian flow from an extending wall with partial slip and Prasad et al. [28] for Casson non-Newtonian boundary layer heat transfer from a cylinder. The mass (solutal) slip phenomenon (in addition to velocity slip) was recently addressed by Bhattacharyya [29] for reactive mass transfer in a porous medium, in which it was shown that mass slip decreases mass transfer rate from the wall and, in addition, also depresses the concentration magnitude.

In the present study, we examine, for the first time, momentum and mass slip in the rotating mass transfer (species diffusion) and boundary layer flow from a spinning cone in an 
anisotropic porous medium. An axisymmetric laminar steady-state formulation with the generalized anisotropic Darcy law is used. The governing transport equations are rendered dimensionless and solved subject to modified boundary conditions with Maple17 software. The solutions are found to correlate well with Chebyshev spectral collocation computations for the general model and with earlier non-porous, no-slip solutions from the literature.

\section{Mathematical Model}

The geometry of the problem is depicted in Figure 1, with respect to an $(x, y, \theta)$ coordinate system. Steady-state, laminar, incompressible, axisymmetric, natural convective mass transfer in boundary layer flow from a rotating porous cone embedded in an anisotropic saturated high-permeability medium is examined. The diffusing species is non-reactive. Rotation is sufficiently weak to neglect compressibility effects. An anisotropic Darcy model is employed to simulate different permeabilities in the medium, which is homogenous and fullysaturated. Large permeabilities are considered which simulate foam-like materials. Forchheimer drag (inertial) and Brinkman boundary vorticity effects are ignored. The $X$ direction is orientated along the cone slant surface, the $Y$ direction normal to this and $\theta$ designates the angle in a plane perpendicular to the vertical symmetry axis:

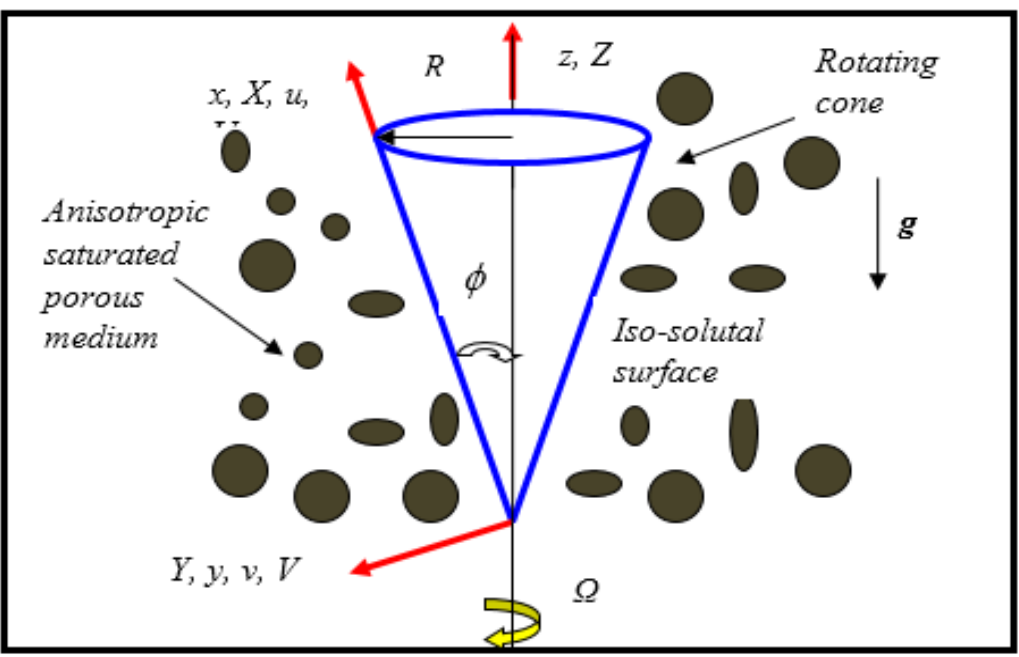

Fig. 1: Physical model for convective mass transfer from a rotating cone in an anisotropic porous medium

The conservation equations for the problem may be presented by amalgamating the models in Bég et al. [26] (for heat and mass diffusion), Wang [27] (for anisotropic slip effects), Prasad et al. [28] (for anisotropic porous media drag forces and thermal slip) and Ece [31] (for the convective mass transfer analogy and rotational body forces in spinning flow) and take the form:

Mass:

$\frac{\partial(R U)}{\partial X}+\frac{\partial(R V)}{\partial Y}=0$ 
X-Momentum:

$U \frac{\partial U}{\partial X}+V \frac{\partial U}{\partial Y}-W^{2} \frac{(R)^{\prime}}{R}=v \frac{\partial^{2} U}{\partial Y^{2}}-\frac{v}{K_{X}} U+g \beta \cos \phi\left(C-C_{\infty}\right)$

$\theta$-Momentum:

$U \frac{\partial W}{\partial X}+V \frac{\partial W}{\partial Y}+U W \frac{(R)^{l}}{R}=v \frac{\partial^{2} W}{\partial Y^{2}}-\frac{v}{K_{\theta}} W$

Species (Concentration):

$U \frac{\partial C}{\partial X}+V \frac{\partial C}{\partial Y}=D \frac{\partial^{2} C}{\partial Y^{2}}$

The Boussinesq approximation has been used so that buoyancy effects only appear in the $X$ direction momentum equation (2), which is coupled to the species diffusion equation, constituting a free convective mass transfer regime. Since the medium is anisotropic, two discrete permeabilities are featured in the $X$ and $\theta$ direction momentum equations. These permeabilities appear as denominators in the Darcian bulk drag force terms in eqns. (2) and (3). A complex set of boundary conditions are prescribed at the surface and far from the cone, following Fang and Jing [30]:

$U=N_{1} v \frac{\partial U}{\partial Y}, V=-\frac{D}{(X / L)\left(1-C_{w}\right)}\left(\frac{\partial C}{\partial Y}\right), W=\Omega R+N_{1} v \frac{\partial W}{\partial Y}, C=C_{\infty}+\left(C_{w}-C_{\infty}\right)(X / L)+D_{1} \frac{\partial C}{\partial Y}$ at $Y=0$,

$U=W=0 ; C=C_{\infty}$ as $Y \rightarrow \infty$.

The velocity components are related to velocity gradients and species gradients, and furthermore the species boundary condition is linked to a species concentration gradient. In the formulation of [30], "slip factors" can then be easily introduced which allows the correct simulation of slip effects without the need to perform molecular dynamics simulations at the wall and instead permits the incorporation of local slip effects in the framework of boundarylayer theory. Since mass slip is being studied i.e. slip of solute, a mass slip boundary condition must be included at the wall. Slip is not just confined to hydrodynamic or thermal slip. Mass slip is a separate phenomenon characterizing polymer and petroleum flows in spin coating. Here the following notation applies: $X$ - coordinate parallel to cone surface, $Y$-coordinate normal to cone surface, $\theta$-angular coordinate, R-radial coordinate, $U$ - velocity component in $X$ direction, $\mathrm{V}$-velocity component in $\mathrm{Y}$ direction, W-velocity component in $\theta$ direction, C-fluid concentration, $\mathrm{C}_{w}$-cone surface concentration, $\mathrm{C}_{\infty}$-free stream concentration, $\mathrm{U}^{*}$ - reference velocity, g-gravitational acceleration, $v$-kinematic viscosity of fluid, $\rho$-density of fluid, $\mathrm{Kx}$ permeability in $\mathrm{X}$ direction, $\mathrm{K}_{\theta}$-permeability in $\theta$ (tangential coordinate) direction, $\mathrm{D}$ - thermal diffusivity of the fluid, $\beta$-coefficient of mass expansion of the fluid, $\Omega$-rotational velocity of the cone (spin velocity about symmetry axis), $\phi$-semi-vertex angle of cone. The primitive boundary layer equations (1) to (4), although strongly nonlinear, may be solved by numerical methods 
such as a finite difference technique. However important dimensionless numbers can only be invoking by normalization of these equations. Therefore, a group of transformations is defined, as follows:

$$
\begin{aligned}
& x=X / L, y=Y /\left(L G r L^{-1 / 4}\right), r=R / r^{*}, u=U / U^{*}, v=V /\left(U^{*} G r L^{-1 / 4}\right), w=W /(\Omega L), \\
& D a_{x}=K_{x} U r /(v L), D a_{\theta}=K_{\theta} U r /(v L), S c=v / D, U^{*}=\left(g \beta \operatorname{Cos} \phi L\left(C_{w}-C_{\infty}\right)\right)^{-1 / 2}, \\
& \Phi=\left(C-C_{\infty}\right) /\left(C_{w}-C_{\infty}\right), G r=\left(U^{*} L / v\right)^{2}, \operatorname{Re}=\Omega L^{2} / v
\end{aligned}
$$

Here $r^{*}$ is local radius of the cone, $L$ is a reference length (cone slant length).

The transport equations are thereby reduced to the following dimensionless equations:

Mass:

$\frac{\partial(r u)}{\partial x}+\frac{\partial(r v)}{\partial y}=0$

Tangential Momentum

$u \frac{\partial u}{\partial x}+v \frac{\partial u}{\partial y}-\frac{\operatorname{Re}^{2}}{G r} w^{2} / r=\frac{\partial^{2} u}{\partial y^{2}}-\frac{u}{D a_{x}}+\Phi$

\section{Swirl Momentum}

$u \frac{\partial w}{\partial x}+v \frac{\partial w}{\partial y}+u w / r=\frac{\partial^{2} w}{\partial y^{2}}-\frac{w}{D a_{\theta}}$

Concentration (Species):

$u \frac{\partial \Phi}{\partial x}+v \frac{\partial \Phi}{\partial y}=\frac{1}{S c} \frac{\partial^{2} \Phi}{\partial y^{2}}$

The boundary conditions are now rendered into the following form:

$u=a \frac{\partial u}{\partial y}, v=-\frac{D\left(C_{w}-C_{\infty}\right) v}{2 x\left(1-C_{w}\right) U^{*} L G r^{-1 / 4}} \frac{\partial \Phi}{\partial y}, w(x, 0)=r+a \frac{\partial w}{\partial y}, \Phi=x+b \frac{\partial \Phi}{\partial y}$ at $y=0$

$u=w=0 ; \Phi=0$ as $y \rightarrow \infty$.

In Eqns. (8)-(13), the following notation applies: F-similarity boundary layer stream function, Gsimilarity boundary layer rotational (swirl) velocity, $\mathrm{H}$-similarity boundary layer temperature function, $x$-transformed $X$ coordinate, $y$-transformed $Y$ coordinate, $r$-transformed local cone radius, $u$-transformed $X$ velocity, $v$-transformed $Y$ velocity, w-transformed $\theta$ velocity, Daxtangential Darcy number, Da $a_{\theta}$ (swirl Darcy number), Sc-Schmidt number, $\Phi$-non-dimensional concentration function, Gr-species Grashof number, Re-rotational Reynolds number. The nondimensional equations (9) to (12) can be further simplified by employing appropriate similarity transformations. We first define a dimensional stream function, $\psi$, following Ece [31]:

$$
r u=\frac{\partial \psi}{\partial y}, r v=-\frac{\partial \psi}{\partial x}
$$

The boundary layer variables are now re-scaled as follows, with $r=x \sin \phi$ (where $r$ is dimensionless local radius of the cone):

$\psi(x, y)=x r F(y), w=r G(y), \Phi=x H(y)$ 
Introducing these relations into Eqns. (8)-(13), generates the following system of "self-similar "ordinary differential equations, with the mass conservation eqn. (8) being automatically satisfied:

Tangential Momentum:

$F^{\prime \prime \prime}+2 F F^{\prime \prime}-\left(F^{\prime}\right)^{2}-\frac{F^{\prime}}{D a_{x}}+N_{R} G^{2}+H=0$

Swirl Momentum:

$G^{\prime \prime}+2 F G^{\prime}-2 F^{\prime} G-\frac{G}{D a_{\theta}}=0$

Species Diffusion (Concentration):

$\frac{1}{S c} H^{\prime \prime}+2 F H^{\prime}-F^{\prime} H=0$

where $F$ is the boundary-layer stream function, $G$ is the boundary-layer rotational (swirl) velocity, $H$ is the boundary-layer concentration, $\kappa_{x}$ and $\kappa_{\theta}$ denote the $x$-direction and $\theta$-direction Darcy numbers, $N_{R}=(\operatorname{Re} \sin \varphi)^{2} / G r$ is the rotational (spin) parameter, $a=N_{1} v / L G r^{-1 / 4}$ is the velocity (momentum) slip parameter, $B=\frac{D\left(C_{w}-C_{\infty}\right) v}{\left(1-C_{w}\right) U^{*} L G r^{-1 / 4}}$ is the cone surface suction/injection parameter, $b=D_{1} / L G r^{-1 / 4}$ is the mass (concentration) slip parameter. For the purely fluid case, $D a_{x}$ (tangential Darcy number) $\rightarrow \infty$ and $D a_{\theta}$ (swirl Darcy number) $\rightarrow \infty$ since the permeability of the anisotropic regime becomes infinite (vanishing fibers of the porous medium). For the case of an isotropic porous medium, $D a_{x}=D a_{\theta}$. The self-similar momentum equations (16) and (17) then reduce to exactly the non-magnetic case of the generalized hydromagnetic equations solved by Ece [31]:

$$
\begin{aligned}
& F^{\prime \prime \prime}+2 F F^{\prime \prime}-\left(F^{\prime}\right)^{2}+N_{R} G^{2}+H=0 \\
& G^{\prime \prime}+2 F G^{\prime}-2 F^{\prime} G=0
\end{aligned}
$$

Eqn. (18) is identical also to the similarity heat transfer equation solved by Ece [31], although in that study the variable $H$ designates temperature, not concentration. The transformed boundary conditions for the current problem take the form:

$F=\frac{S}{2 S c} H^{\prime} ; F^{\prime}=a F^{\prime \prime} ; G=1+a G^{\prime}, H=1$ at the cone surface $(y=0)$

$F^{\prime} \rightarrow 0 ; G \rightarrow 0 ; H \rightarrow 0$ in the freestream $(y \rightarrow \infty)$

\section{Numerical Solutions by Maple 17}

The self-similar nonlinear two-point boundary value problem defined by eqns. (16)-(18) under boundary conditions (21) has been solved using MAPLE17 [32] shooting quadrature, employing Runge-Kutta-Fehlberg methods. This code has been utilized in many viscous 
boundary layer flow problems recently by the authors including nanofluid slip flows in magnetic fields [33], Marangoni biological convection [34] and rheological nanofluid convection in porous media [35]. A Runge-Kutta-Fehlberg fourth-fifth order numerical algorithm (RKF45) is employed, available in the symbolic computer software Maple 17. This utilizes a collocation method in which a finite-dimensional space of candidate solutions is selected (usually, polynomials up to a certain degree) with a number of points in the domain (called collocation points), in order to generate a stable, accurate and fast converging solution which satisfies the given equation at the collocation points. The RFK45 algorithm is adaptive since it adjusts the quantity and location of grid points during iteration and thereby constrains the local error within acceptable specified bounds. In the current problem, the asymptotic boundary conditions given in Eq. (21) are replaced by a finite value in the range 10-15 depending on the parameter values. Infinity is prescribed to ensure that all numerical solutions approach the asymptotic values correctly. The selection of sufficiently large value for infinity is imperative for maintaining desired accuracy in boundary layer flows, and is a common pitfall encountered in numerous studies. The stepping formulae used to solve Eqns. (16)-(18) under conditions (21) via fifth-fourth order Runge-Kutta-Fehlberg algorithms are given below [36]:

$$
\begin{aligned}
& k_{0}=f\left(x_{i}, y_{i}\right) \\
& k_{1}=f\left(x_{i}+\frac{1}{4} h, y_{i}+\frac{1}{4} h k_{0}\right) \\
& k_{2}=f\left(x_{i}+\frac{3}{8} h, \quad y_{i}+\left(\frac{3}{32} k_{0}+\frac{9}{32} k_{1}\right) h\right) \\
& k_{3}=f\left(x_{i}+\frac{12}{13} h, \quad y_{i}+\left(\frac{1932}{2197} k_{0}-\frac{7200}{2197} k_{1}+\frac{7296}{2197} k_{2}\right) h\right) \\
& k_{4}=f\left(x_{i}+h, \quad y_{i}+\left(\frac{439}{216} k_{0}-8 k_{1}+\frac{3860}{513} k_{2}-\frac{845}{4104} k_{3}\right) h\right) \\
& k_{5}=f\left(x_{i}+\frac{1}{2} h, \quad y_{i}+\left(-\frac{8}{27} k_{0}+2 k_{1}-\frac{3544}{2565} k_{2}-\frac{1859}{4104} k_{3}-\frac{11}{40} k_{4}\right) h\right) \\
& y_{i+1}=y_{i}+\left(\frac{25}{216} k_{0}+\frac{1408}{2565} k_{2}+\frac{2197}{4104} k_{3}-\frac{1}{5} k_{4}\right) h \\
& z_{i+1}=z_{i}+\left(\frac{16}{135} k_{0}+\frac{6656}{12825} k_{2}+\frac{28561}{56430} k_{3}-\frac{9}{50} k_{4}+\frac{2}{55} k_{5}\right) h
\end{aligned}
$$

Here $y$ denotes fourth-order Runge-Kutta phase and $z$ is the fifth-order Runge-Kutta phase. An estimate of the error is achieved by subtracting the two values obtained. If the error exceeds a specified threshold, the results can be re-calculated using a smaller step size. The approach to estimating the new step size is shown below:

$$
h_{\text {new }}=h_{\text {old }}\left(\frac{\varepsilon h_{\text {old }}}{2\left|z_{i+1}-y_{i+1}\right|}\right)^{1 / 4}
$$




\section{Validation with Chebyshev Spectral Collocation}

The fifth order boundary value problem defined by eqns. (16)-(18) with wall and free stream boundary conditions (21) has also been solved with a spectral collocation method of Chebyshev type (SCM), using a Matlab-based code, SPINCHEB, developed for rotating transport phenomena. Spectral methods can provide exponential convergence in space and have been widely used to solve nonlinear fluid mechanics problems. Although these methods are relatively infrequently applied to simulate multi-dimensional complex geometries, for simple geometries e.g. channels, they provide excellent accuracy [37]. This technique has also been implemented to successfully simulate many diverse problems in applied thermomechanics including unsteady radiation convection in cavities [38], thermal conduction in biological materials [39], electrohydrodynamic (EHD) ion drag pumping flows at general electrical Hartmann numbers [40], magneto-hydrodynamic blood flow in a curved tube (magnetized Dean flow) [41] and nonlinear magnetic propulsion flows [42]. The principal advantage of SCM lies in the accuracy achievable for a given number of unknowns. For problems for which the solutions are sufficiently smooth, SCM demonstrates exponential rates of convergence and accuracy. To optimize the spectral solutions for the present problem, SPINCHEB has been tested for convergence with respect to the spatial resolution. The solutions converge in 30 iterations with Newton's method. Numerical solutions are found to be independent of the number of collocation points for a sufficiently large number of collocation points. $N=70$ yields the optimal convergence and very high accuracy (up to $10^{-6}$ ) and is therefore implemented in all the computations in SPINCHEB. In SCM, we seek an approximate solution, which is a global Chebyshev polynomial of degree $N$ defined on the remapped interval $[-1,1]$. We discretize the interval by using collocation points to define the Chebyshev nodes in $[-1,1]$, namely:

$$
x_{j}=\cos \left(\frac{j \pi}{N}\right), \quad j=0.1,2, \ldots . . N .
$$

The derivatives of the functions at the collocation points are given by:

$$
f^{n}\left(x_{j}\right)=\sum_{j=0}^{N} d_{k j}^{n} f\left(x_{j}\right), \quad n=1,2 \text {. }
$$

where $d_{k j}^{n}$ represents the differentiation matrix of order $n$ and these are given by:

$$
\begin{aligned}
d^{1}{ }_{k j} & =\frac{4 \gamma_{j}}{N} \sum_{n=0}^{N} \sum_{l=0, n+l=o d d}^{n-1} \frac{n \gamma_{n}}{c_{l}} T_{l}^{n}\left(x_{k}\right) T_{n}\left(x_{j}\right), \quad k, j=0,1, \ldots . N, \\
d^{2}{ }_{k j} & =\frac{2 \gamma_{j}}{N} \sum_{n=0}^{N} \sum_{l=0, n+l=\text { even }}^{n-2} \frac{n \gamma_{n}\left(n^{2}-l^{2}\right)}{c_{1}} T_{l}^{n}\left(x_{k}\right) T_{n}\left(x_{j}\right), \quad k, j=0,1, \ldots . N,
\end{aligned}
$$

Here $T_{n}\left(x_{j}\right)$ are the Chebyshev polynomial and the coefficients $\gamma_{j}$ and $c_{l}$ are defined as:

$$
T_{n}\left(x_{j}\right)=\cos \left(n \cos ^{-1} x_{j}\right), \quad \gamma_{j}=\left\{\begin{array}{c}
\frac{1}{2} \quad j=0, \text { or } N \\
1 \quad j=1,2, \ldots . . N-1
\end{array} \quad c_{l}=\left\{\begin{array}{c}
2 \quad l=0, \text { or } N \\
1 \quad l=1,2, \ldots . N-1
\end{array}\right.\right.
$$


As described above, the Chebyshev polynomials are defined on the finite interval $[-1,1]$. Therefore, to apply the Chebyshev spectral method to the nonlinear boundary eqns. (9)-(10), we make a suitable linear transformation and transform the physical domain $[0, \infty]$ to the Chebyshev computational domain $[-1,1]$. We sample the unknown function $w$ at the Chebyshev points to obtain the data vector, $w=\left[w\left(x_{0}\right), w\left(x_{1}\right), w\left(x_{2}\right), \ldots \ldots w\left(x_{N}\right),\right]^{T}$. The next step is to find a Chebyshev polynomial $P$ of degree $N$ that interpolates the data, i.e., $P\left(x_{j}\right)=w_{j}, \quad j=0,1, \ldots N$, and obtain the spectral derivative vector $w$ by differentiating $P$ and evaluating at the grid points, i.e., $w_{j}^{\prime}=P^{\prime}\left(x_{j}\right), j=0,1, \ldots N$. This transforms the nonlinear differential equations into a system of nonlinear algebraic equations which are solved by Newton's iterative method starting with an initial guess. We have compared the MAPLE17 solutions, and SPINCHEB computations with the reduced model in [31] obtained when porous drag forces are neglected $\left(D a_{x} \rightarrow \infty\right.$ and $D a_{\theta} \rightarrow \infty$ i.e. vanishing permeability case), the cone is solid $(S=0)$ and mass and velocity slip ignored $(a=b=0)$ and with Schmidt number replacing Prandt number in Ece's heat conservation equation [31]. This reduced system of ordinary differential equations is:

$$
\begin{aligned}
& F^{\prime \prime \prime}+2 F F^{\prime \prime}-\left(F^{\prime}\right)^{2}+N_{R} G^{2}+H=0 \\
& G^{\prime \prime}+2 F G^{\prime}-2 F^{\prime} G=0 \\
& \frac{1}{S c} H^{\prime \prime}+2 F H^{\prime}-F^{\prime} H=0 \\
& F(0)=0 ; F^{\prime}(0)=0 ; G(0)=1, H(0)=1 \text { at the cone surface }(y=0) \\
& F^{\prime}(y) \rightarrow 0 ; G(y) \rightarrow(0) ; H(y) \rightarrow 0 \text { in the freestream }(y \rightarrow \infty)
\end{aligned}
$$

Both MAPLE and SPIN-CHEB correlate very closely with the infinite permeability solutions of [31], as observed in Tables 1 and 2. Confidence in both algorithms is high; however, we elect to present graphical solutions here with MAPLE 17 software.

\section{Maple Computations, Results and Discussions}

Extensive computations are conducted to simulate the variation of the tangential velocity $\left(F^{\prime}\right)$, swirl velocity $(\mathrm{G})$ and concentration $(\mathrm{H})$ with distance, $y$, into the boundary layer (transverse to the cone surface); 7 physical parameters are analyzed i.e. $D a_{x}$-tangential Darcy number, $D a_{\theta}$-swirl Darcy number, $S c$-Schmidt number, $N_{R}$ - rotational (spin) parameter (a function of rotational Reynolds number), $a=N_{1} v / L G r^{-1 / 4}$-momentum (velocity slip) parameter, parameter, $b=D_{1} / L G r^{-1 / 4}$ i.e. mass slip parameter and $S$ - wall mass flux (transpiration) parameter ( $>0$ for suction, $<0$ for injection and $=0$ for solid cone). The regime has high permeability in both the $x$ - and $\theta$-directions so that high values are prescribed for $D a_{x}$ and $D a_{\theta}$. i.e. 0.5 , unless otherwise stated. $S c$ is prescribed as $0.22 . N_{R}=1.0$ (Coriolis force and buoyancy force effects are equivalent) unless otherwise indicated. All computations 
were conducted with MAPLE17 and are illustrated in Figures 2-23. Infinity is prescribed as 12 to ensure asymptotically smooth solutions are attained in the free stream.

In Figures 2-5, the effects of rotational parameter, $N_{R}=(\operatorname{Re} \operatorname{Sin} \phi)^{2} / G r$ on tangential and swirl velocities, temperature and Nusselt number function (cone surface temperature gradient) are presented. In these figures, when $D a_{x}=D a_{\theta}$ the regime is isotropic. Inspection of Figure 2 shows that with greater values of spin parameter, $N_{R}$, the tangential velocity is boosted significantly, in particular in close proximity, to the cone surface. This parameter arises only in the tangential momentum eqn. (16) as a quadratic assistive body force term, $+N_{R} G^{2}$. This couples the swirl momentum equation (17) very strongly to the tangential momentum field and leads to a marked influence of the former on the latter. As elaborated, this effect is assistive to the tangential velocity near the cone surface and for some distance into the boundary layer, transverse to the cone surface. However, as we progress further from the cone, the trend is infact reversed. The re-distribution of axial momentum via swirl near the cone is strongest in the regime closest to the axis of rotation; however further towards the free stream the effect diminishes and leads to a fall in tangential velocities (and corresponding increase in axial momentum, not shown). Momentum boundary layer thickness is accentuated initially with greater spin parameter i.e. larger Coriolis force associated with greater rotational cone velocity $(\Omega)$ enhances rotational Reynolds number ( $\left.\operatorname{Re}=\Omega L^{2} / v\right)$, which increases the spin parameter $\left(N_{R}=(\operatorname{Re} \sin \varphi)^{2} / G r\right)$, for a prescribed cone semi-apex angle and species Grashof number (Gr). Of course, the present study is laminar- with sufficiently greater rotational Reynolds numbers, flow transition and indeed separation will arise. Therefore, to maintain adherence of the boundary layer to the cone surface and mitigate detachment, the cone rotation must be constrained within certain limits. This avoids centrifugal instability as elaborated by Hussain [43], although he only considers viscous flow i.e. his model neglects species diffusion. In Figure. 2 the tangential velocity magnitudes are always positive indicating that flow reversal is never induced. The damping of tangential velocity with greater suction effect is also apparent in Figure 2. The rotating boundary layer is drawn closer to the cone surface with negative $S$ values. The reverse effect is associated with greater surface injection (positive $S$ ) for which much higher tangential velocities are computed. For both cases, asymptotically smooth decay of the tangential velocity into the free stream is achieved. Swirl flow is slightly depressed (Figure 3 ) close to the cone surface. The swirl velocity profiles descend sharply in this vicinity. implying that swirl momentum boundary layer thickness is slightly increased in this zone. The freestream vanishing swirl velocity is attained very quickly. The swirl momentum which is lost to the tangential field with greater rotational velocity of the cone manifests in a depletion in swirl velocities in the fluid. These results agree with the trends reported strongly by Salzburg and Kezios [16], one of the relatively few studies which has considered purely convective mass transfer from a spinning cone. Greater suction $(S<0)$ at the cone surface further acts to depress swirl velocity whereas enhanced injection $(S>0)$ accelerates the swirl flow and 
decreases momentum boundary layer thickness. Concentration of the diffusing species, $C$, (Figure 4) is substantially decreased with increasing $N_{R}$, however the decay is much more gradual from the cone surface to the free stream. Greater spin effect therefore depresses concentration (species) boundary layer thickness. Suction suppresses mass diffusion in the flow whereas injection results in a boost in mass diffusion i.e. elevation in concentration magnitudes. In consistency with figure 4 , the mass transfer rate (dimensionless Sherwood number function) magnitudes (Figure 5) are elevated near the cone surface with greater spin parameter. The reduction in concentrations in the species boundary layer is induced by an elevation in mass flux to the cone surface. With suction, the mass transfer rate is increased whereas with injection it is reduced.

Figures 6-9 present the evolution of the flow and species characteristics with tangential Darcy number, $D a_{\theta}$ i.e. $\theta$-direction permeability function. With greater $D a_{\theta}$ (and these values are intentionally large owing to the sparsely packed nature of the porous medium considered), the tangential velocity, $F^{\prime}$, is noticeably elevated near the cone surface (Figure 6), attaining a peak and thereafter plummeting to the vanishing free stream value. This behaviour is sustained through the boundary layer for all values of transverse coordinate with all profiles converging in the free stream. The tangential flow is therefore accelerated strongly with greater $D a \theta$ values. In the swirl momentum equation, the inverse proportionality of the Darcian bulk impedance to tangential Darcy number is evident in the drag force term, $-\left(G / D a_{\theta}\right)$, which has the opposite effect to the Coriolis body force. The coupling of the swirl and tangential momenta equations manifests in a strong influence of this Darcian drag impedance on the tangential flow. As tangential permeability increases, the porous medium comprises a progressively lower volume of solid particles (which cause resistance) and becomes increasingly fluidic in nature. In chemical filtration processes, greater velocity control is therefore achieved with lower permeabilities in the tangential direction. The porous medium may be designed therefore to capitalize on this effect. Increasing injection at the cone surface physically accelerates the tangential flow whereas increasing suction suppresses it. Swirl velocity, G, (Figure 7) although also enhanced with greater tangential Darcy number, is less dramatically altered than tangential velocity. The swirl velocity field is also damped out much faster than the tangential field- profiles decay sharply to the free stream value for any value of rotation parameter even the case of a stationary cone $\left(N_{R}=0\right)$. Irrespective of the value of $D a \theta$, the wall suction is observed to decelerate swirl flow whereas injection generates the converse response. As with the tangential flow, backflow does not arise for even high suction values. With increasing $D a \theta_{\theta}$, concentration ( $H$ ) values (Figure 8 ) are markedly elevated throughout the boundary layer. The wall values are also strongly increased. Increasing permeability decreases the concentration of solid particles in the regime i.e. increases the presence of voids. This serves to provide greater fluid for the species to diffuse in and enhances concentration distributions. With greater wall suction $(S<0)$ the species diffusion (mass transfer) is suppressed whereas it is encouraged with wall injection $(S>0)$. The momentum 
boost with injection also serves to exacerbate species diffusion, owing to the coupling of the tangential flow with the species diffusion field via eqns. (16) and (18) respectively. In these figures, Schmidt number, $S c$, is assumed to be 0.22 which corresponds to hydrogen diffusing in air [44], a common scenario in chemical engineering processing. Figure 9 demonstrates that the Sherwood number function (dimensionless mass transfer rate i.e. $H$ ) at the wall is considerably reduced with increasing tangential Darcy number and surface injection; it is as expected, elevated with decreasing Darcy number and surface suction.

Figures 10-13 depict the effects of swirl Darcy number, $D a_{x}$ i.e. $x$-direction permeability function, on the flow and mass transfer characteristics. A dramatic acceleration in the tangential velocity is sustained with increasing swirl Darcy number (figure 10); this effect is generally consistent at all values of transverse coordinate. In the tangential momentum equation (16) the $D a_{x}$ parameter arises in the Darcian retarding force term, - $F^{\prime} / D a_{x}$, in a fashion analogous to the presence of $D a_{\theta}$ in the drag force term, $-\left(G / D a_{\theta}\right)$ in eqn. (17). Anisotropy is achieved in the porous medium when $D a_{x} \neq D a_{\theta}$. This provides an alternate approach to that propounded by Storesletten and Rees [45] wherein an anisotropic permeability ratio is employed, preventing individual variation of hydraulic conductivity properties in different directions in terms of a discrete Darcy number. Increasing $D a_{x}$ evidently reduces the tangential Darcian drag force which will effectively accelerate tangential flow. A noteworthy acceleration in tangential flow is also induced by strong injection at the cone with a retardation in the flow corresponding to an increase in suction parameter $(S<0)$. In Figure 11 we note that a non-trivial deceleration in swirl flow is computed with large increase in $D a_{x}$, since the gain in tangential momentum is compensated for by a fall in swirl momentum. Strong enhancement in swirl flow however accompanies increasing injection at the wall with strong deceleration resulting from large suction. Concentration, $H$, is significantly reduced (Figure 12) with greater swirl Darcy number, $D a_{x}$. As indicated earlier with progressive increase in permeability, the regime comprises a lower quantity of solid material fibers; this provides a greater volume of fluid enabling tangential momentum to be elevated and swirl momentum to be stifled. The latter results in suppression of species diffusion with greater swirl Darcy number values which manifests in a thinning in the species boundary layer thickness. Injection at the cone surface however is found to accelerate swirl flow whereas suction decelerates it. Surface concentration gradient $\left(H^{\prime}\right)$ i.e. Sherwood number function, is enhanced in magnitude with increasing swirl Darcy number (Figure 13) in particular, in close proximity to the cone surface. The flux of species away from the body of rotating fluid (boundary layer) to the wall therefore depresses concentration boundary layer thickness.

Figures 14-16 present the influence of Schmidt number $(S c)$ on the velocity functions and concentration function distributions. A disproportionate number of studies address heat transfer in rotating cone flow compared with mass transfer. Schmidt number is therefore rarely studied in the literature for such flows. This important mass transfer parameter symbolizes the 
ratio of the momentum to the mass diffusivity in convective mass transfer processes. It effectively quantifies the relative effectiveness of momentum and mass transport by diffusion in the hydrodynamic (velocity) and concentration (species) boundary layers. Smaller Sc values can represent for example hydrogen gas as the species diffusing ( $S c \sim 0.2$ ). $S c=1.0$ corresponds to both momentum and species boundary layer thicknesses being the same. In the present investigation we consider $S c<1$, for species diffusivity exceeds momentum diffusivity and this range is appropriate for low-molecular weight gases (e.g. Hydrogen, Helium) diffusing in air. With increasing $S c$, the tangential velocity (Figure 14) is very strongly decelerated; the near-wall peak is also progressively displaced closer to the cone surface with increasing Schmidt number. As noted earlier, tangential velocity is always greater for injection at the wall compared with suction. Concentration, $H$, as expected, is markedly suppressed throughout the boundary layer regime (Figure 15), with an increase in Sc. Maximum $H$ corresponds to the lowest $S c$ value (0.22) since species diffusivities are much higher for such cases. Mass transfer rates i.e. Sherwood number function values (Figure 16) are elevated in magnitude, in particular, very close to the cone surface with an increase in Schmidt number. However, with greater injection mass transfer rate magnitudes are depressed whereas with stronger suction they are enhanced. Larger Schmidt numbers therefore achieve a consistent contraction in the concentration boundary layer. With thinner concentration boundary layers, the concentration gradients will be enhanced causing a decrease in concentration of species in the boundary layer. The implication for chemical engineering designers is that in such a regime, a diffusing species with a lower Schmidt number is more amenable to achieving enhanced concentration distributions in the porous medium. This has also been noted by Gebhart et al. [46].

Figures 17-20 show the effects of the momentum slip parameter (a) on velocity functions and concentration characteristics. These plots correspond to very strong anisotropy with a much greater permeability in the swirl direction compared with the tangential direction. Figure 17 shows that for the injection case $(S>0)$ there is a consistent enhancement in tangential velocity at the wall owing to the slip effect and that this increases the near-wall peak velocity and also results in a migration in peaks closer to the wall. With increasing suction, a near-wall peak velocity is only computed when momentum slip is present- it is absent for the no-slip case $(a=0)$. The tangential velocities as described earlier are accelerated with injection and retarded with suction. Significant acceleration in the swirl velocity, G, close to the wall is also observed with greater momentum slip in Figure 18. In all cases the maximum swirl velocity occurs at the cone surface and thereafter decays smoothly to zero in the free stream i.e. there are no overshoots in velocity near the wall. Injection always achieves greater magnitudes in swirl velocity than suction. The momentum boundary layer thickness is decreased as a result of increasing momentum slip at the wall. Figures 19 and 20 reveals that concentration values and Sherwood number function values are respectively increased in magnitude with greater momentum slip and greater wall injection (mass flux into the boundary layer) whereas they are 
reduced in magnitude with greater wall suction (removal of fluid from the boundary layer through the cone surface).

Finally, Figures 21-23 illustrate the influence of the solutal (concentration) slip parameter, $b$, on the convective mass transfer characteristics. Tangential velocity (Figure 21) is strongly decelerated with greater mass slip effect for any value of injection parameter. However, the tangential velocity is observed to be boosted with increasing $b$ values when suction at the cone surface is present. Magnitudes of tangential velocity are however greater with injection than with suction. Figure 22 shows that species concentration is consistently decreased at the wall (cone surface) whether injection or suction is present, with an increase in mass slip parameter. Greater concentration is however achieved in the boundary layer with injection relative to suction. Both the momentum (hydrodynamic) slip and mass slip effects are executed via the cone surface boundary conditions, as documented in eqn. (21). They clearly alter the velocity and concentration fields in a substantial manner. Concentration gradient (Sherwood number function) is conversely increased with greater mass slip, due to the diffusion of species away from the boundary layer towards the cone surface. Greater wall suction enhances mass transfer rates whereas injection results in the converse effect.

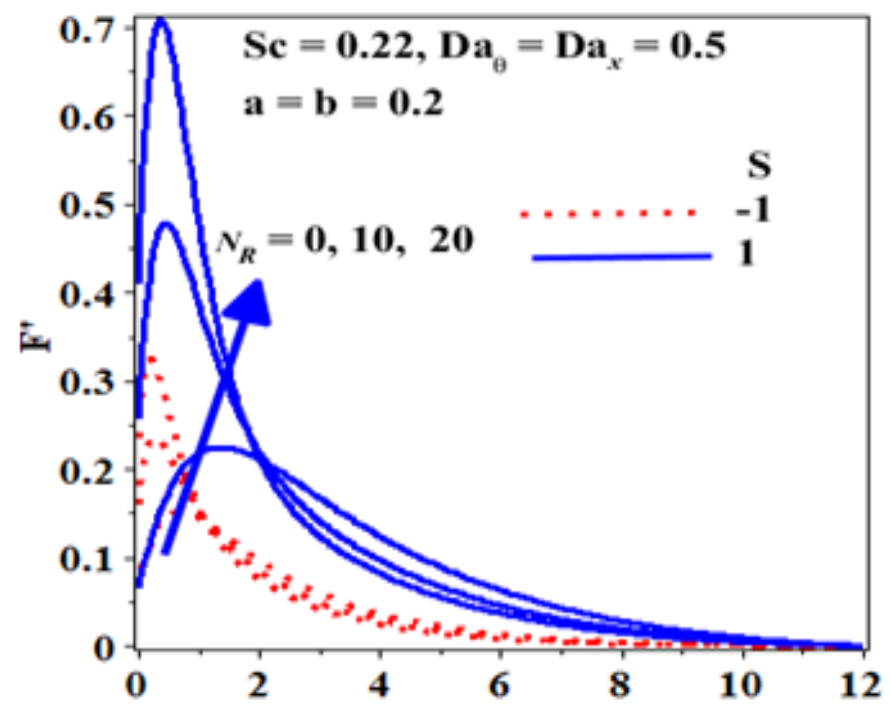

Fig 2: Tangential velocity $(F)$ for various $N_{R}$ 


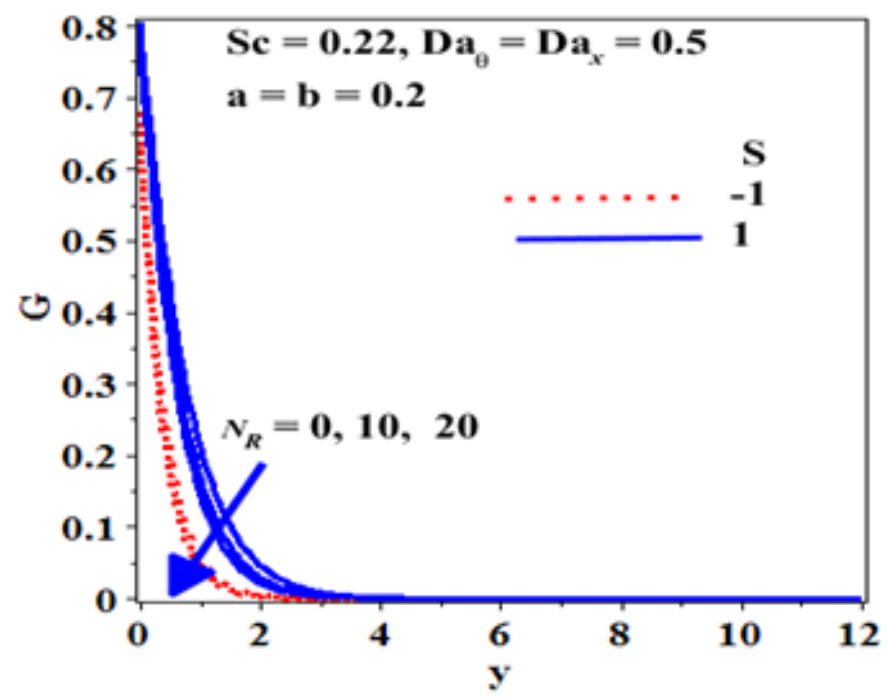

Fig. 3: Swirl velocity (G) for various $N_{R}$.

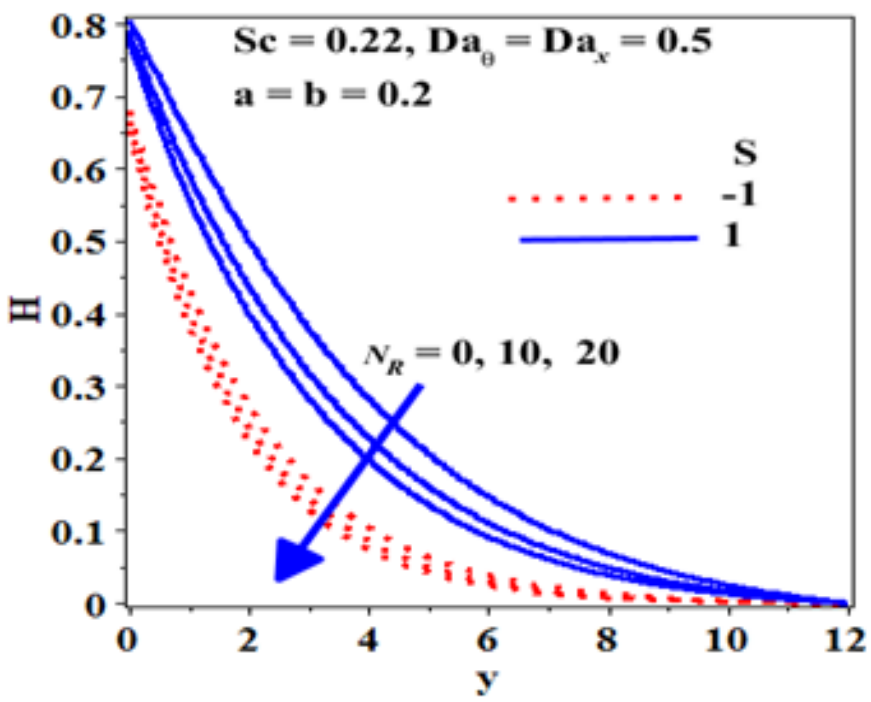

Fig. 4: Concentration $(H)$ for various $N_{R}$.

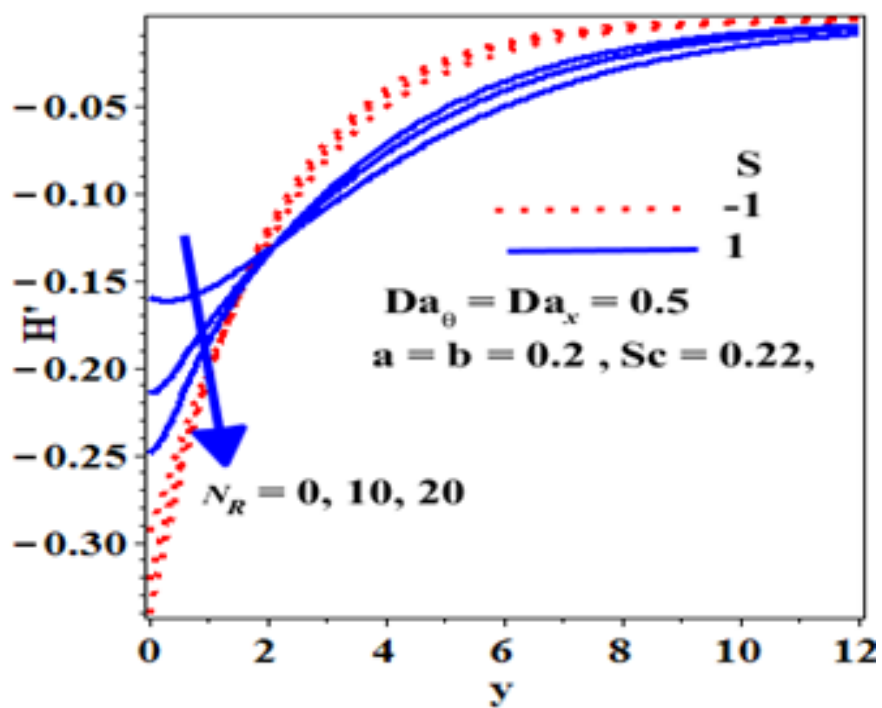

Fig. 5: Concentration gradient $(H)$ for various $N_{R}$. 


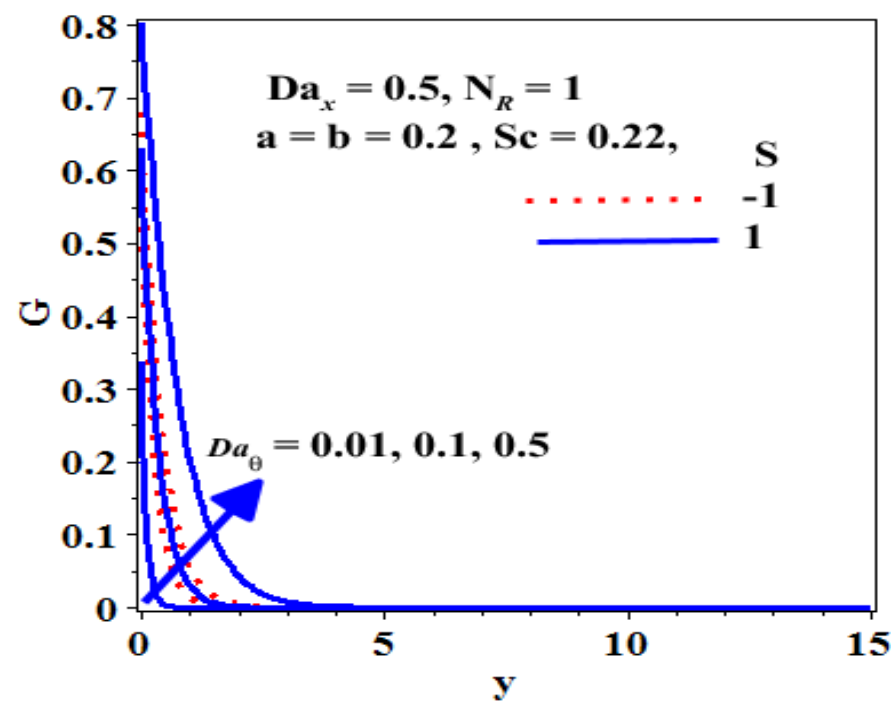

Fig. 6: Tangential velocity $(F)$ for various $D a \theta$.

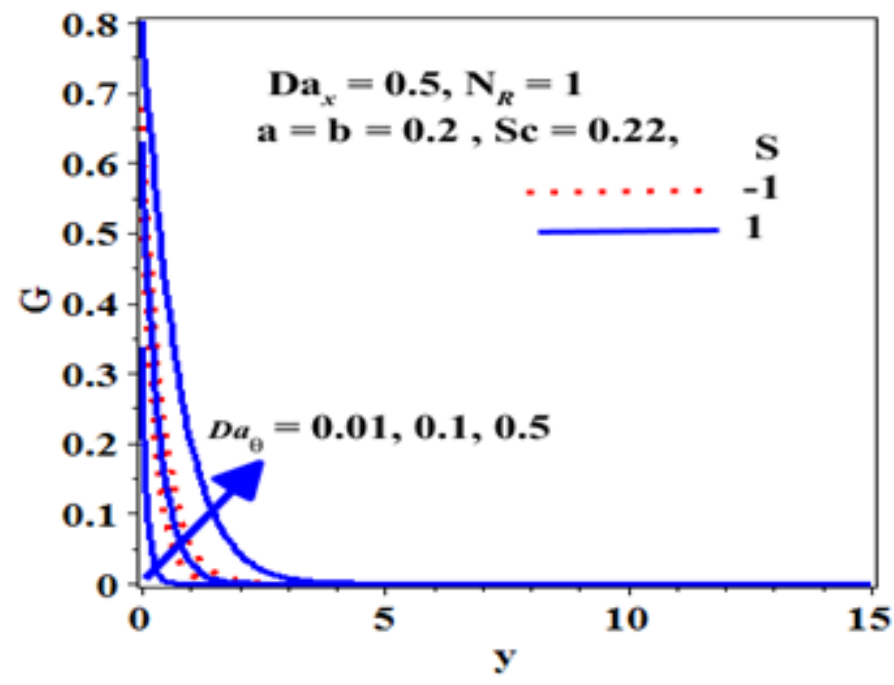

Fig. 7: Swirl velocity (G) for various $D a_{\theta}$.

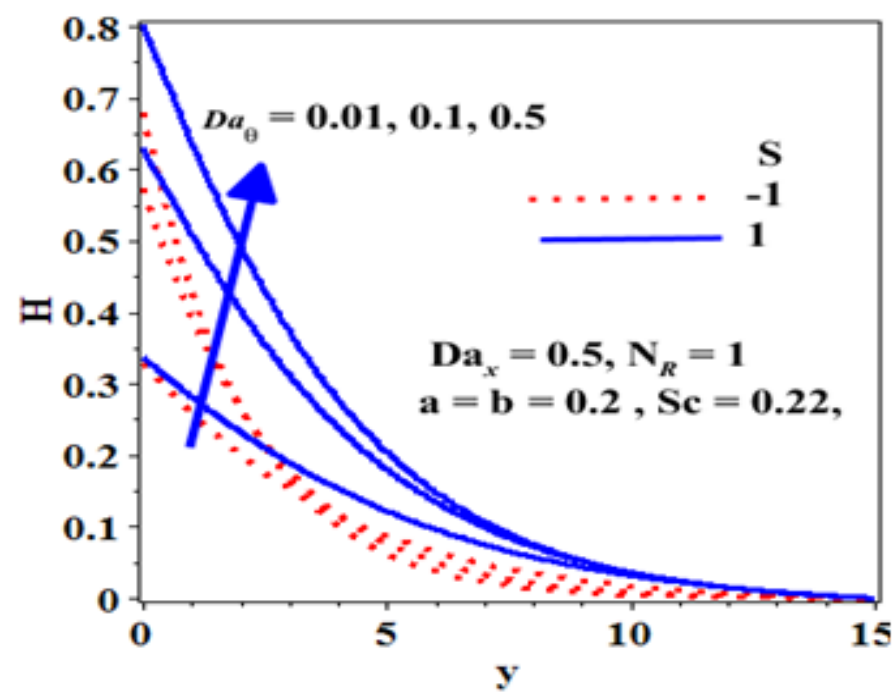

Fig. 8: Concentration $(H)$ for various $D a_{\theta}$. 


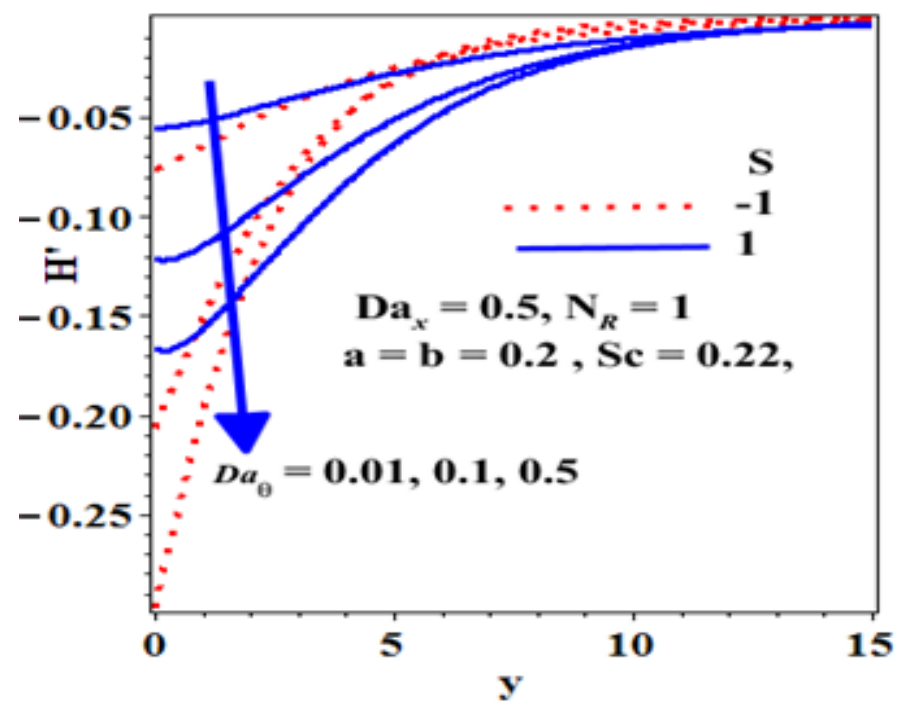

Fig. 9: Concentration gradient $(H)$ for various $D a \theta$.

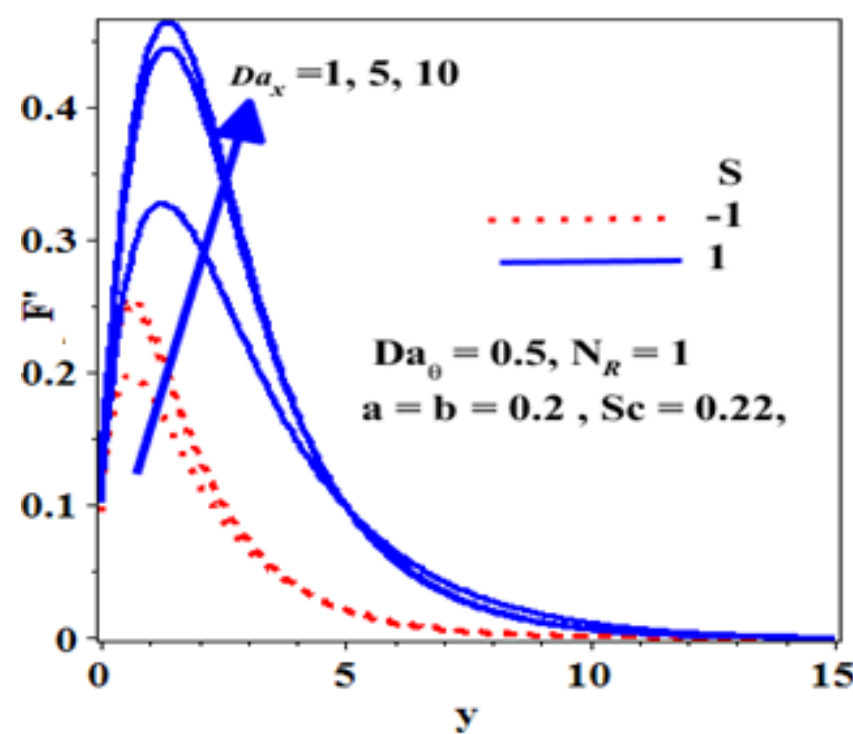

Fig. 10: Tangential velocity $(F)$ for various $D a_{x}$.

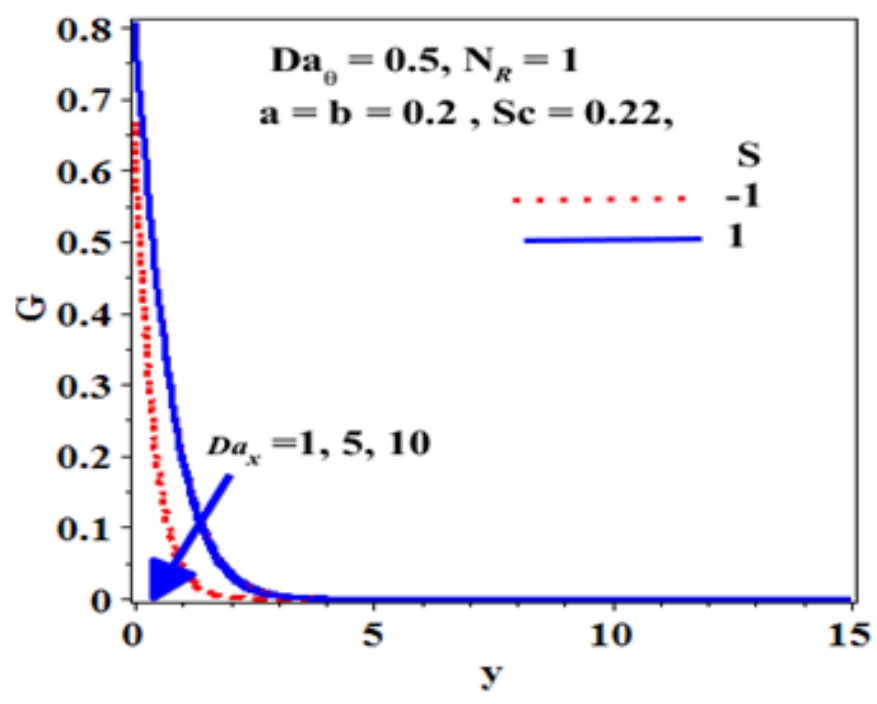

Fig. 11: Swirl velocity $(G)$ for various $D a_{x}$. 


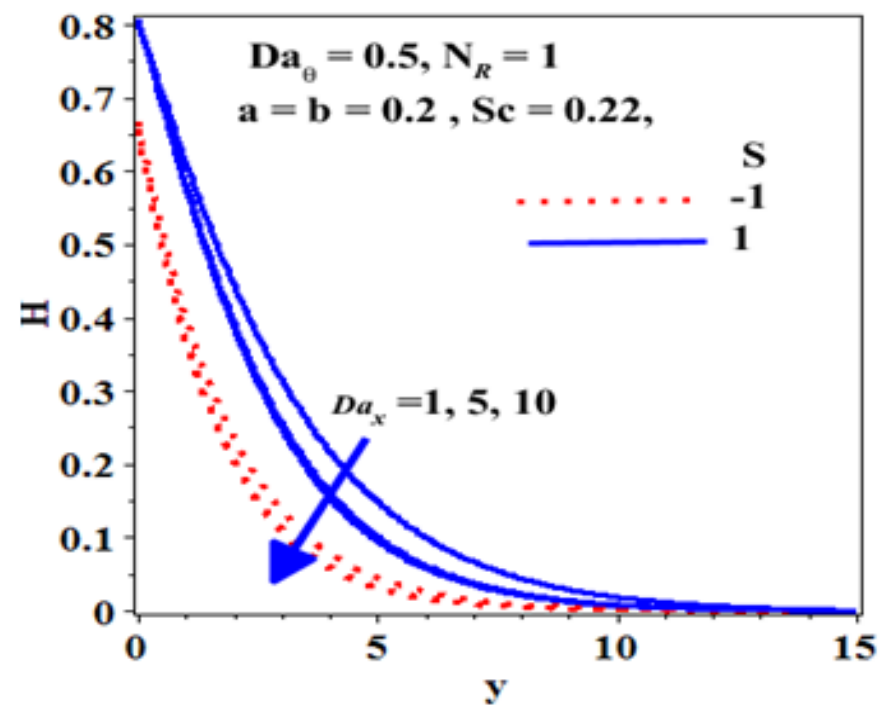

Fig. 12: Concentration $(H)$ for various $D a_{x}$.

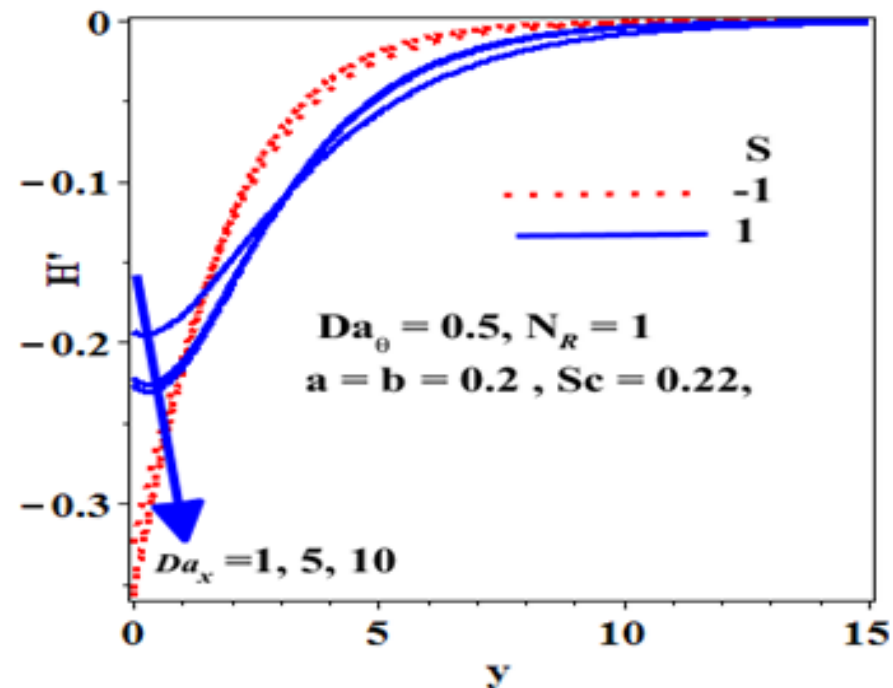

Fig. 13: Concentration gradient $(H)$ for various $D a_{x}$.

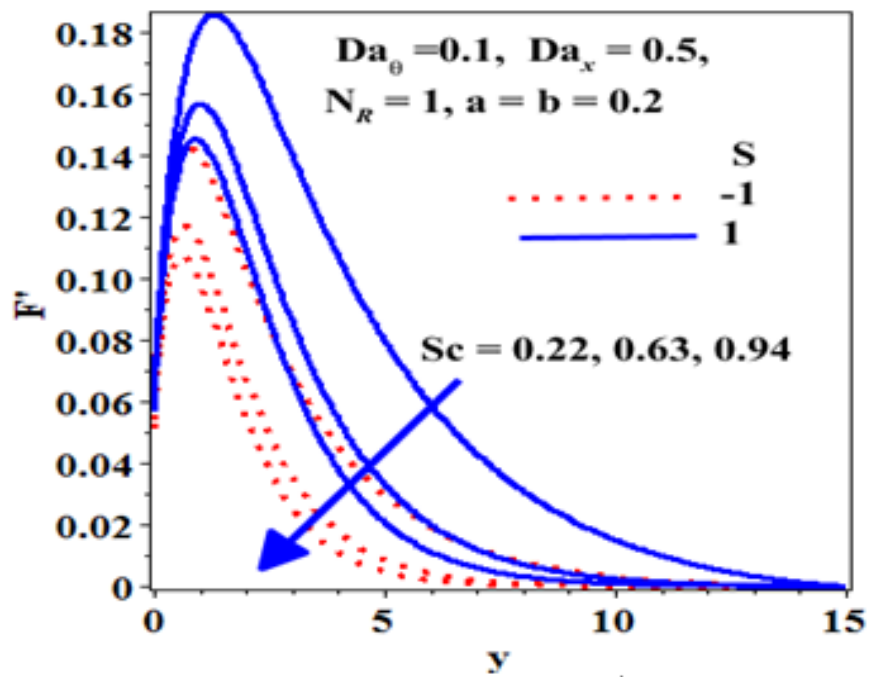

Fig. 14: Tangential velocity $(F)$ for various SC. 


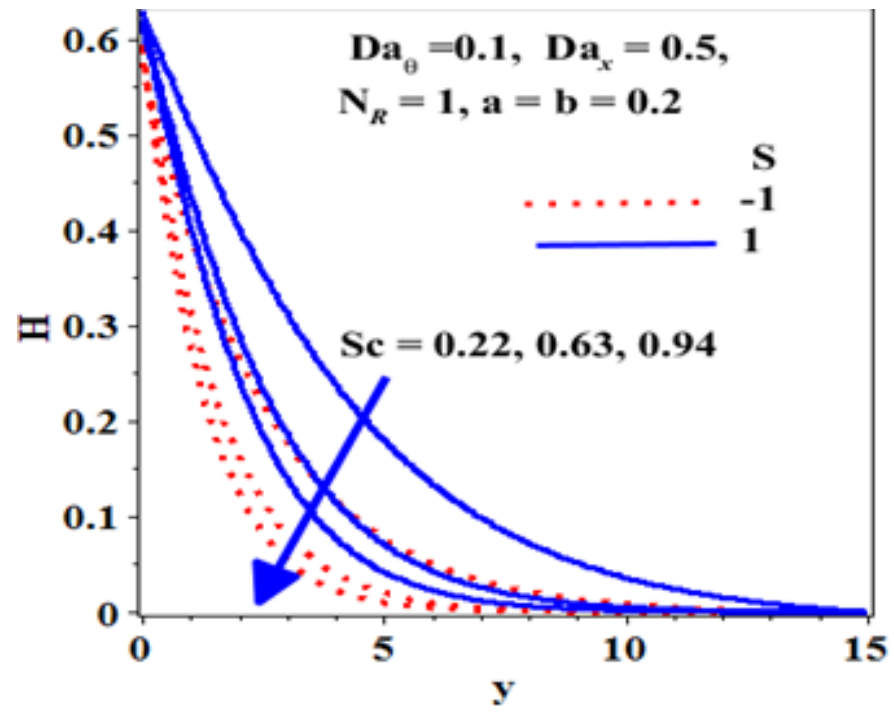

Fig. 15: Concentration $(H)$ for various $S c$

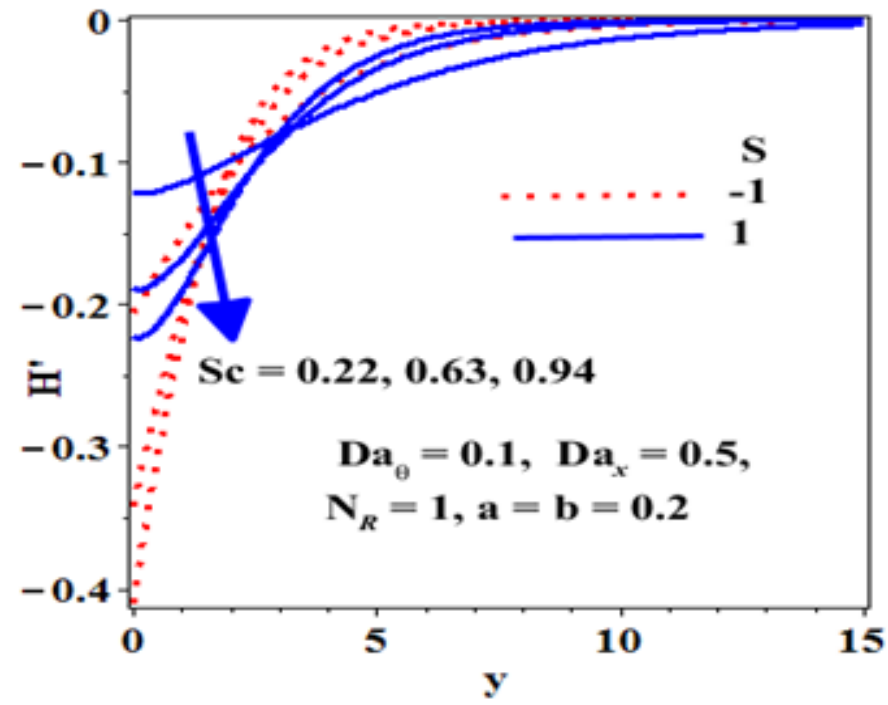

Fig. 16: Concentration gradient $(H)$ for various Sc.

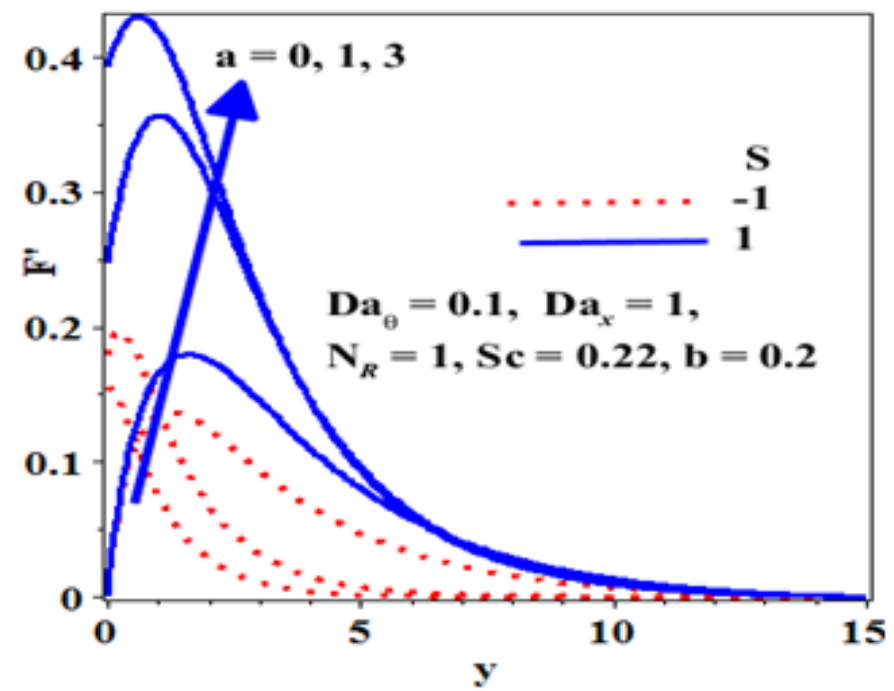

Fig 17: Tangential velocity $(F)$ for various a. 


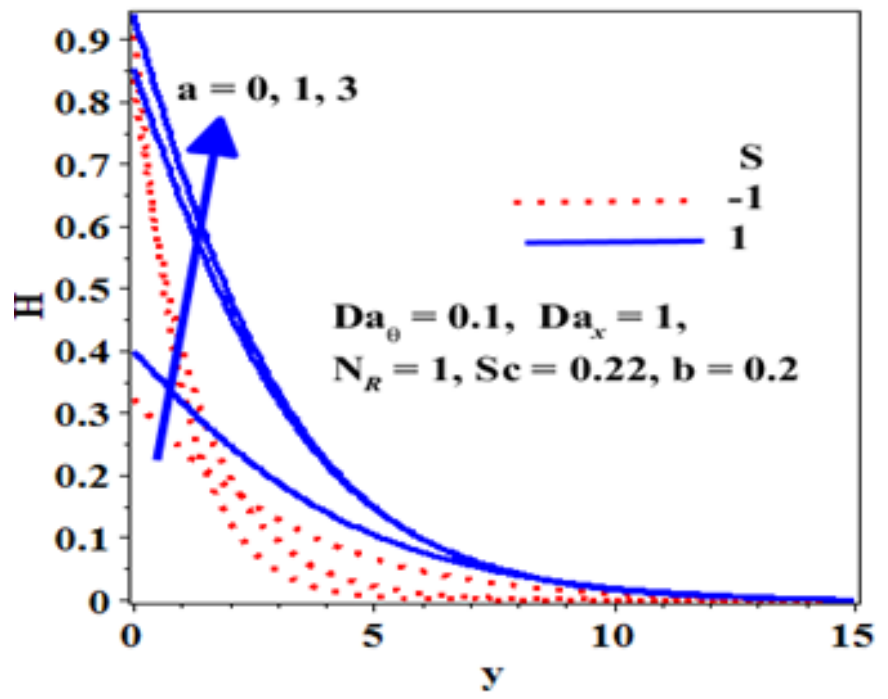

Fig. 18: Swirl velocity $(G)$ for various a.

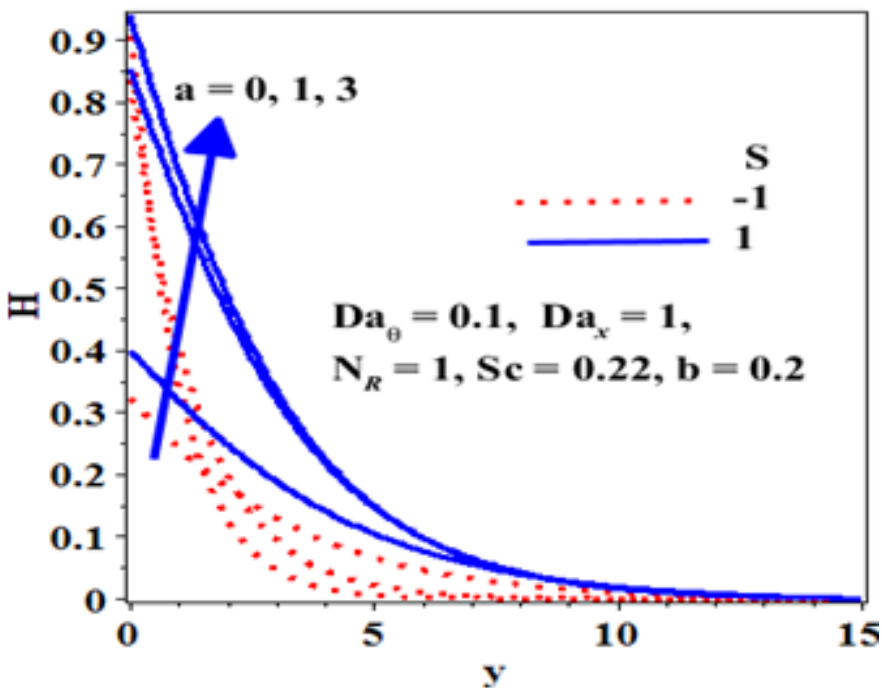

Fig. 19: Concentration $(H)$ for various $a$.

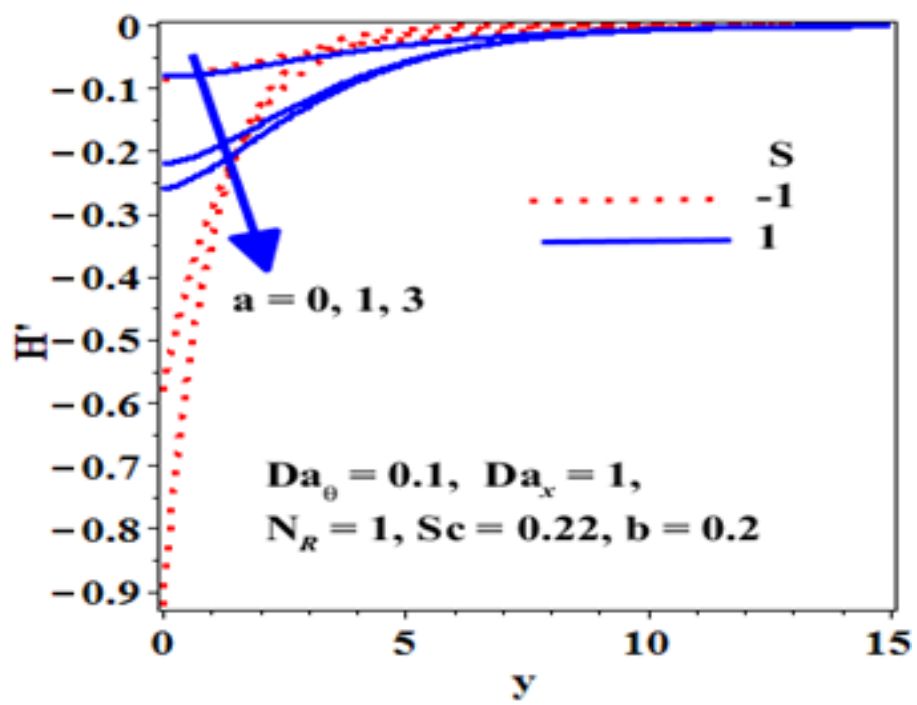

Fig. 20: Concentration gradient $(H)$ for various a. 


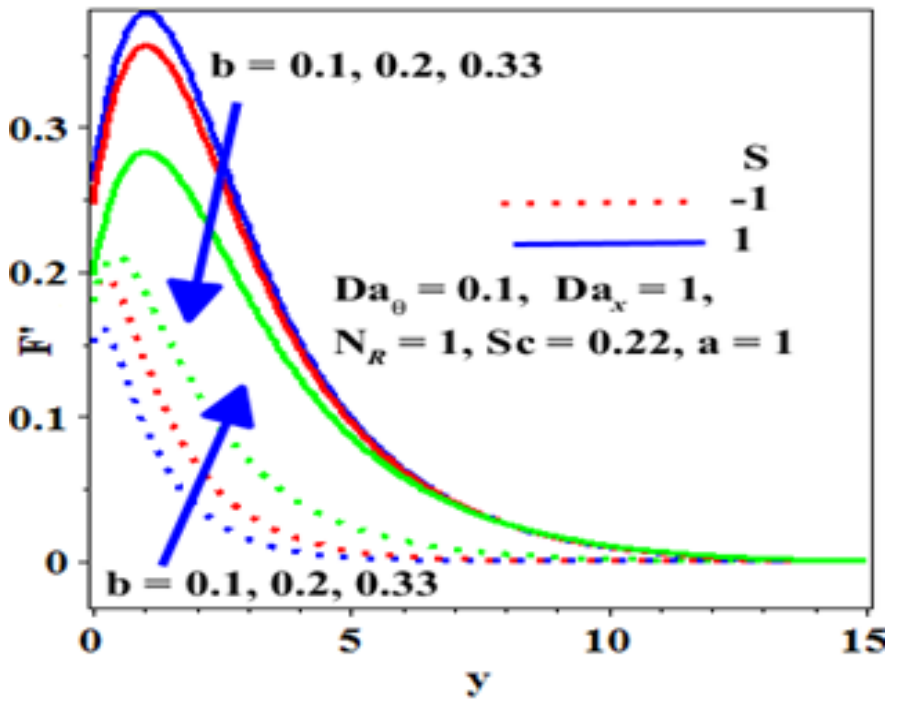

Fig 21: Tangential velocity $(F)$ for various $b$

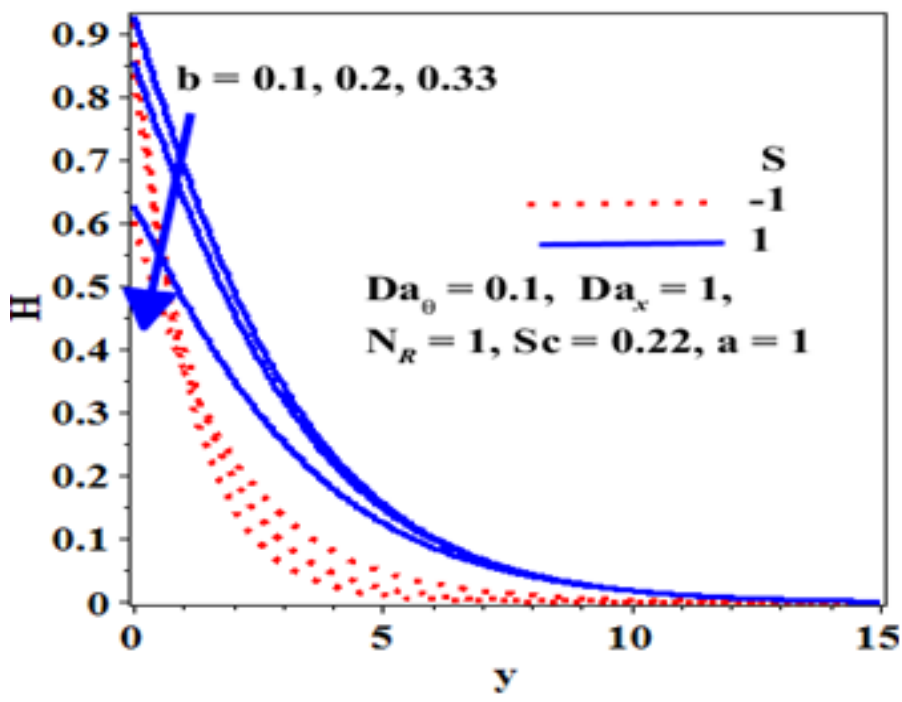

Fig. 22: Concentration $(H)$ for various $b$.

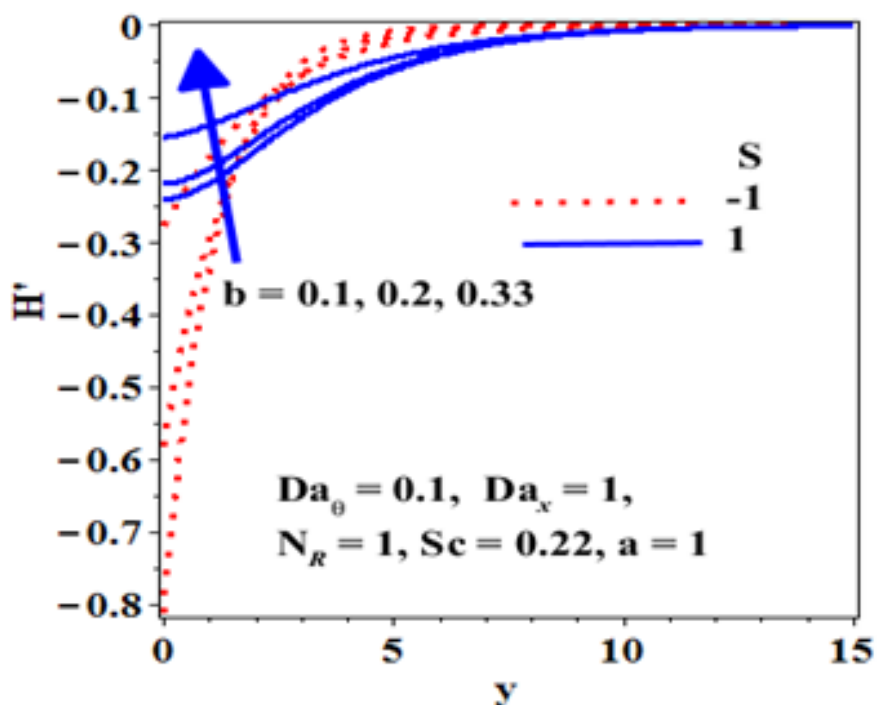

Fig. 23: Concentration gradient $(H)$ for various $b$. 
Tables 1 and 2 further indicate that with an increase in spin parameter $\left(N_{R}=(\operatorname{Re} \sin \varphi)^{2} / G r\right)$, the primary skin friction (tangential velocity gradient) i.e. $\left(F^{\prime \prime}\right)$ is elevated whereas with an increase in Schmidt number it is depressed. The near the surface of rotation is forced radially outwards with a simultaneous upward flow induced tangential to the spinning body surface. $N_{R}=(\operatorname{Re} \sin \varphi)^{2} / G r$ and this parameter relates inertial forces to buoyancy forces. As this parameter increases the tangential momentum is boosted and this accelerates the tangential flow leading to a rise in primary (tangential) skin friction. The increase in Schmidt number induces the contrary effect. The concentration boundary layer thickness is substantially lower than velocity (momentum) boundary layer thickness. The thickening of the momentum boundary layer corresponds to a deceleration in the flow which explains the fall in tangential velocity gradient i.e. tangential surface shear stress function $\left(F^{\prime \prime}\right)$ with larger Schmidt numbers.

Table 1: Values of $\mathrm{F}^{\prime \prime}(0)$ for free-convective mass transfer over a spinning cone with $\mathrm{Dax} \rightarrow \infty$ and $\mathrm{Da}_{\theta} \rightarrow \infty$ (purely fluid case) and injection/suction and mass/momentum slip ignored.

\begin{tabular}{|l|l|l|l|l|l|l|}
\hline \multirow{2}{*}{$N_{R}$} & \multicolumn{5}{|c|}{$S c=1$} & \multicolumn{3}{c|}{$S c=10$} \\
\cline { 2 - 7 } & \multicolumn{3}{|c|}{$F^{\prime \prime}(0)$} \\
\cline { 2 - 7 } & Ece [31] & SPIN-CHEB & Maple 17 & $($ Ece, 2006) & SPIN-CHEB & Maple 17 \\
\hline 0.0 & 0.68150212 & 0.6814901 & 0.6814833 & 0.43327726 & 0.43298991 & 0.4291876 \\
\hline 0.5 & 0.84650616 & 0.8464791 & 0.8464882938 & 0.62601869 & 0.62458713 & 0.6228014 \\
\hline 1.0 & 1.00196008 & 1.00194601 & 1.001943127 & 0.79828572 & 0.79839769 & 0.798418104 \\
\hline 2.0 & 1.29230021 & 1.29228503 & 1.29228491 & 1.10990481 & 1.10990489 & 1.10990496 \\
\hline
\end{tabular}

Table 2: Values of $-H^{\prime}(0)$ for free-convection mass transfer over a spinning cone with $\mathrm{Dax} \rightarrow$ $\infty$ and $\mathrm{Da}_{\theta} \rightarrow \infty$ (purely fluid case) and injection/suction mass/momentum slip.

\begin{tabular}{|l|l|l|l|l|l|l|}
\hline \multirow{2}{*}{$N_{R}$} & \multicolumn{5}{|c|}{$-H^{\prime}(\mathrm{O})$} \\
\cline { 2 - 7 } & \multicolumn{5}{|c|}{$S c=1$} & \multicolumn{3}{c|}{$S c=10$} \\
\cline { 2 - 7 } & Ece [31] & SPIN-CHEB & Maple 17 & $($ Ece, 2006) & SPIN-CHEB & Maple 17 \\
\hline 0.0 & 0.63886614 & 0.63885988 & 0.63885470 & 1.27552680 & 1.268971 & 1.26598645 \\
\hline 0.5 & 0.67194897 & 0.67194396 & 0.67193844 & 1.47165986 & 1.504325 & 1.547638332 \\
\hline 1.0 & 0.70053401 & 0.70052873 & 0.70052453 & 1.60768499 & 1.607697 & 1.60756132 \\
\hline 2.0 & 0.74869559 & 0.74869028 & 0.74868824 & 1.80575019 & 1.805749 & 1.80574943 \\
\hline
\end{tabular}

\section{Conclusions}

A self-similar mathematical model has been derived for the steady free convective mass transfer from a rotating porous cone embedded in an anisotropic Darcian highly permeable medium with surface momentum and mass (solutal) effects. The partial differential boundary layer equations have been transformed into a system of coupled, nonlinear ordinary differential equations, by virtue of a group of appropriate scaling transformations. A complex set of boundary conditions have been imposed at the cone surface. MAPLE 17 quadrature numerical solutions have been obtained for the seventh order nonlinear two-point boundary value problem. Validation has been achieved with earlier non-porous solutions in the absence of mass and momentum slip (Ece [31]) and additionally via a Chebyshev spectral collocation algorithm, achieving very good correlation. 
Anisotropy has been studied via a unique Darcy number in each momentum conservation equation. Tangential and swirl velocities have been found to be generally elevated with increasing permeability functions (i.e. $\theta$ and $x$-direction Darcy parameters). Increasing spin velocity of the cone has been shown to accelerate tangential flow but to decelerate swirl flow. Greater suction is also found to retard both tangential and swirl flow whereas increasing injection manifests in the converse effect. With greater momentum slip, both tangential and swirl flows are accelerated. Concentration values and Sherwood number function values are also enhanced with momentum slip but only for the case of wall injection. A strong reduction in tangential velocity is generated with greater mass (solutal) slip effect for any value of injection parameter. Concentration is also depressed at the wall (cone surface) whether injection or suction is present, with an increase in mass slip parameter. Generally, the parametric investigation performed has demonstrated that spin coating processes in chemical engineering can be influenced strongly with simple modifications in the porous medium anisotropy, cone surface conditions (slip effects) and in the judicious selection of the diffusing species. The present model has been confined to Newtonian flow. However, in spin coating operations many liquids may exhibit non-Newtonian (rheological) characteristics. Future studies may therefore examine rotating convective mass transfer of nonNewtonian fluids with slip effects and interesting possible rheological models include micropolar viscoelastic models [48], Jeffery viscoelastic nanofluids [49] (which also involve thermal diffusion), variable-viscosity models [50] and second order Reiner-Rivlin differential models which approximate well the behavior of certain polymeric coatings [51]. Additionally, it may be useful to explore the combined heat and mass transfer in spin coating flows with the inclusion of nonFourier heat transfer models [52] in conjunction with non-Fickian mass transfer models.

\section{Acknowledgements}

The Authors wish to express their cordial thanks to Reviewers for their valuable suggestions and constructive comments to improve the quality of the paper.

\section{References}

[1] L Bennamoun and A Belhamri, Fluid Dynamics and Material Processing. 4221 (2008)

[2] A S Altevogt, D E Rolston and S Whitaker, Advances in Water Resources. 26717 (2003).

[3] O Anwar Bég and O D Makinde, Petroleum Sci. Eng. 7693 (2011).

[4] C Nicholson, Rep. Prog. Phys. 64(7) 815 (2001).

[5] M A G Ulson de Souza and S Whitaker, Braz. J. Chem. Eng. 20(2) 191 (2003).

[6] K G Helmer, J D Bernard and H S Christopher, NMR in Biomedicine. 8297 (1995).

[7] R M Cotta, C Baohua and P F L Heilbron Filho, Convective Heat and Mass Transfer in Porous Media. NATO ASI Series. 525 (1991).

[8] J Piquemal, F A Kulacki and F Arinç, Transport in Porous Media. 10271 (1993).

[9] K Vafai and C L Tien, Int. J. Heat and Mass Transfer. 251183 (1982).

[10] H Marcus, J. Geophysical Research. 675215 (1962).

[11] X Wang, F Thauvin and K K Mohanty, Chem. Eng. Sci. 541859 (1999).

[12] K L Adams, W B Russel and E Rebenfeld, Int. J. Multiphase Flow. 14203 (1988). 
[13] G Mishra and A Reddy, ASCE J. Hydraul. Eng. 109(6) 897 (1983).

[14] G Chiogna, O A Cirpka, M Rolle and A Bellin, J. Chem. Phys. 51261 (2015).

[15] A Nakayama and F Kuwahara, Hand Book of Porous Media, Editor (K. Vafai), CRC Press, 235 (2005).

[16] F Salzberg and S P Kezios, ASME J. Heat Transfer. 87(4) 469 (1965).

[17] J Newman, J. Electrochem. Soc. 11969 (1972).

[18] K A Smith and C K Colton, AlChemE. J. 18949 (1972).

[19] B T Ellison and I Cornet, J. Electrochem. Soc. 11868 (1971).

[20] C M Mohr and J Newman, J. Electrochem. Soc. 1231687 (1976).

[21] M Toren, M Ungarish, G Pinchuk and A Solan, ASME. J. Appl. Mech. 58(2) 566 (1991).

[22] A A Rashaida, D J Bergstrom and R J Sumner, ASME J. Appl. Mech. 73(1) 108 (2005).

[23] Y Taamneh and R Omari, J. Fluids. 2013 60483-1 (2013).

[24] A F Miguel, Thermal Sci. 16(1) 167 (2012).

[25] W A Khan, M J Uddin and A I M Ismail, PloS one 8(3) e54024 (2013).

[26] O Anwar Bég, M J Uddin, M M Rashidi and N Kavyani, J. Eng. Thermophysics. 23(2) 79 (2014).

[27] C Y Wang, Chem. Eng. Sci. 573745 (2002).

[28] V R Prasad, A Subba Rao, N B Reddy, B Vasu and O Anwar Bég, Proc. IMechE-PART E: J. Proc. Mech. Eng. 227(4) 309 (2013).

[29] K Bhattacharya, Front. Heat Mass Transfer. 3043006 (2012).

[30] T Fang and W Jing, Commun. Nonlinear Sci. Numer. Simult. 193086 (2014).

[31] M C Ece, Appl. Math. Comput. 179231 (2006).

[32] MAPLE from Waterloo Maple Software Inc., Canada (www.maplesoft.com) (2015).

[33] M J Uddin, O Anwar Bég and N S Amin, J. Magn. Mag. Mat. 368252 (2014).

[34] O Anwar Bég, M Ferdows, S Islam and M N Islam, J. Mech. Med. Biol. 14 1450039-1 (2014).

[35] M J Uddin, N H M Yusoff, O Anwar Bég and A I M Ismail, Phys. Scr. 871 (2013).

[36] M J Uddin, W A Khan and A I M Ismail, Transport in Porous Media. 92867 (2012).

[37] J P Boyd, Chebyshev \& Fourier Spectral Methods, 2nd Ed., Dover, New York, USA (2001).

[38] G J Li, J Ma and B W Li, ASME J. Heat Transfer. 137(3) 032701 (2015). https://doi.org/10.1115/1.4029237

[39] A F Elsayed and O Anwar Bég, J. Mech. Med. Biol. 14(3) 1450043-1 (2014).

[40] O Anwar Bég, M Hameed and T A Beg, Int. J. Comput. Meth. Eng. Sci. Mech. 14104 (2013).

[41] M M Hoque, M M Alam, M Ferdows and O Anwar Bég, Proc. IMechE-Part H: J. Eng. Med. 227(11) 1155 (2013).

[42] O Anwar Bég, Numerical methods for multi-physical magnetohydrodynamics, Chapter 1, New Developments in Hydrodynamics Research, September, Nova Science, New York (2012).

[43] Z Hussain, PhD Thesis, Applied Mathematics, University of Birmingham, UK (2010).

[44] B Gebhert, Heat Conduction and Mass Diffusion, MacGraw Hill, New York (1993).

[45] L Storelsetten and D A S Rees, Transport in Porous Media. 1979 (1995).

[46] B Gebhart, Y Jaluria, R L Mahajan and B Sammakia, Buoyancy-Induced Flows and Transport, Hemisphere, Washington, USA (1988).

[47] D Tripathi and O Anwar Bég, Computer Methods in Biomech. Biomed. Eng. 18(15) 1648 (2015).

[48] Z Mehmood, R Mehmood and Z Iqbal, Commun. Theor. Phys. 67(4) 443 (2017).

[49] R Mehmood, S Nadeem, S Saleem and N S Akbar, J. Taiwan Inst. Chem. Eng. 7449 (2017).

[50] R Tabasum, R Mehmood and O Pourmehran, Part E: J. Proc. Mech. Eng. 232(5) 622 (2018).

[51] G Makanda, O D Makinde and P Sibanda, Math. Prob. Eng. 2013934712 (2013).

[52] O D Makinde, N Sandeep, I L Animasaun and MS Tshehla, Defect and Diffusion Forum. 37467 (2017). 